Resumen: La interacción entre las diversas disciplinas es una relación de ida y vuelta, en donde todas ellas se nutren, se definen, re-definen y sus fronteras disciplinares son cada vez más difusas, por esa permanente reconfiguración. Los seres humanos están expuestos a una diversidad de situaciones, inciertas y complejas. Son, justamente, esos desafíos los que permiten ejercitar no sólo la interpretación, sino también la acción para resolverlas, a través de la elaboración de hipótesis de solución y someterlas luego a un proceso de experimentación para contrastar y obtener conclusiones que permitan dar solución a dichos desafíos. Este proceso complejo, no solo cognitivo, sino también de orientación hacia la resolución de una realidad problemática, potencia la agudeza en la observación, la reflexión y la capacidad resolutiva de problemas. A partir de esta reconfiguración, este paper propone la plataforma de pensamiento y acción CITAD o por sus siglas en inglés: SETAD, que transforma la aproximación a la resolución de problemas o identificación de oportunidades, conformando una transdisciplina que reconfigura, modela, experimenta y soluciona la cuestión, al contener en ella, la Ciencia (Science), la Ingeniería (Engineering), la Tecnología (Technology), el Arte (Art) y el Diseño (Design).

Palabras Clave: Diseño - arte - ciencia - ingeniería - tecnología - plataforma - pensamiento - epistemología del diseño.

[Resúmenes en inglés y portugués en la página 122]

${ }^{(1)}$ Oscar Mas. Universidad de Ciencias y Artes de América Latina (UCAL), Lima, Perú. Profesional de perspectiva interdisciplinar en el campo del diseño, la educación, la creatividad, el marketing, el branding y la comunicación. Co-Autor de la metodología Pro. Seso Creativo $^{\varpi}$, de la Universidad de Ciencias y Artes de América Latina. Gestor del FabLab UCAL. Conferencista en creatividad e innovación para diversos foros. Apasionado por el impacto de la educación y el diseño en las personas. 


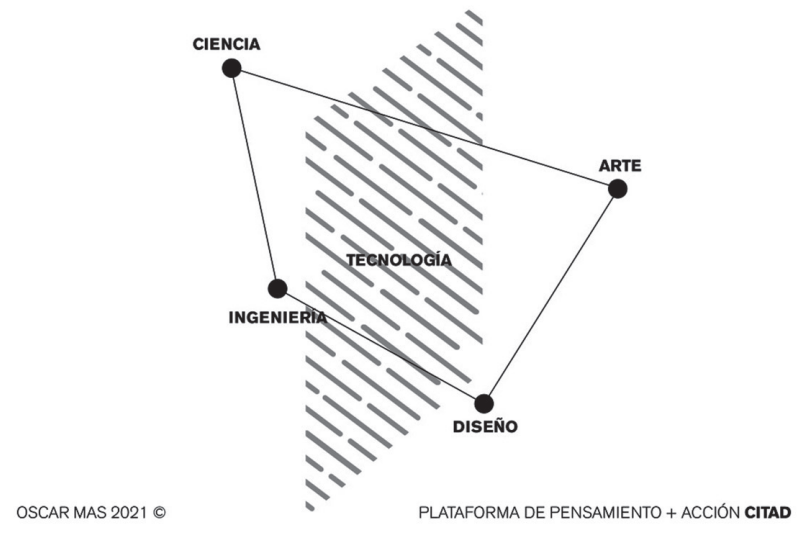

Figura 1. Imagen producida por el autor.

\section{Plataforma de pensamiento y acción CITAD}

El esquema de Rich Gold (2007) expone los conceptos de arte, ciencia, diseño e ingeniería, así como sus diversas interrelaciones y plantea que en el eje 'arte y diseño' se movilizan las mentes, mientras que en el eje 'ciencia e ingeniería' se movilizan las moléculas. En esa misma línea de pensamiento, la combinación entre arte y ciencia sería universal, conformada por conjuntos que establecen patrones, quedando para el duo diseño e ingeniería, la específica, con la finalidad de servir a usuarios y clientes.

En esas interrelaciones del esquema de Gold, se pone de manifiesto la creatividad como una variable que transita entre los ejes y cuya resultante tendría el potencial de innovar. Siguiendo a Gold, la creatividad no es simplemente hacer algo nuevo, sino hacer algo nuevo que represente una nueva categoría, un nuevo género o un nuevo tipo de cosa.

En su libro The Plenitude, Gold sostiene que su trabajo fue diseñar la tapa de un sintetizador y lo que ahora se conoce como 'interfaz de usuario'. En 1971, se conocía como faceplate1 o placa frontal, pues muchos de los sintetizadores parecían equipos médicos. En ese proceso, Gold, mezcla los campos disciplinares de la ingeniería electrónica y las artes plásticas -al experimentar con la visualización de la placa frontal-, la ciencia y, por supuesto, el diseño -como integrador de todos los campos mencionados anteriormente en una placa-. Dicha placa, no solo resulta visualmente amigable, sino que es de fácil comprensión por parte de los usuarios además de ser modificable según sus preferencias, mediante marcadores de colores removibles.

La interacción entre las diversas disciplinas es una relación de ida y vuelta, en donde todas ellas se nutren, se definen, re-definen y sus fronteras disciplinares son cada vez más difusas, por esa permanente reconfiguración. Los seres humanos están expuestos a una diversidad de situaciones, inciertas y complejas y son justamente, esos desafíos los que permiten ejercitar no solo la interpretación, sino también la acción para resolverlas, a través de la elaboración de hipótesis de solución y someterlas luego a un proceso de expe- 
rimentación para contrastar y obtener conclusiones que permitan dar solución a dichos desafíos. Este proceso complejo, no solo cognitivo, sino también de orientación hacia la resolución de una realidad problemática, potencia la agudeza en la observación, la reflexión y la capacidad resolutiva de problemas.

A partir de esta reconfiguración, este paper propone la plataforma de pensamiento y acción CITAD o por sus siglas en inglés: SETAD, que transforma la aproximación a la resolución de problemas o identificación de oportunidades, conformando una transdisciplina que reconfigura, modela, experimenta, soluciona la cuestión, al contener en ella, la Ciencia (Science), la Ingeniería (Engineering), la Tecnología (Technology), el Arte (Art) y el Diseño (Design).

Gold (2007), explica que otra de sus diversas áreas de trabajo, fue la de crear múltiples análisis de lenguajes y síntesis de sistemas. Menciona en sus escritos, el caso de su experimento denominado Nida Structures, basado en el Dr. Nida2, lingüista que lo inspiró para dicho ensayo. En esta experiencia compartida con el autor, le interesaba responder a una pregunta de investigación que trataba de comprobar: es posible construir una estructura de palabras que le diera un sentido al enunciado de manera aleatoria. De esta forma, se evidenciaba la mezcla, la experimentación y las interacciones entre campos disciplinares que generan nuevas posibilidades.

Según Burgos (2009), el arte, la ciencia, la tecnología y el diseño, están relacionados y comparten elementos que los hacen similares. El arte contemporáneo ha alcanzado niveles altísimos de adaptación a las tecnologías digitales y a la utilización de computadoras, así como también la ciencia y la tecnología se valen de procesos y técnicas desarrolladas, en un principio, para ser utilizadas por el área de las artes, al momento de comunicar y visualizar sus contenidos y resultados. (Burgos, 2009 p.98)

Siguiendo a este autor, el arte contemporáneo implica expresiones multidisciplinares que hacen uso de todos aquellos recursos disponibles. La tecnología aporta procesos y metodologías a la ciencia, versus los procesos del arte que son más intuitivos y abstractos. El campo disciplinar del diseño se comporta como conciliador del circuito, aportando a distintos campos por igual.

El diseño y arte actúan de un lado del conocimiento, la ciencia y la tecnología del otro, pero de algún modo, todos están complementándose, ya sea aplicando conocimientos o descubrimientos o quizá tratando de comprender los procesos, principios y fundamentos que los hacen más efectivos para producir mejores resultados en un área específica, y aplicándolos de manera experimental en otras áreas de estudio. (Burgos, 2009, p.103)

Gold (2007) sostiene que en ese intercambio, los artistas pueden trabajar conjuntamente con los científicos, usando la tecnología como un lenguaje común entre pares y sugiere que, una vez que se hayan conformado estos equipos, es pertinente proporcionarles libertad para llegar a la plenitud planteada.

De acuerdo al autor en cuestión, no es necesario que los artistas se conviertan en diseñadores para los proyectos de ciencia, ni que los científicos se conviertan en ingenieros para los artistas, el objetivo es que construyan cosas a partir de un interés en común. En el 
proceso de intercambio, pueden actuar como diseñadores o ingenieros, por una cuestión de roles momentáneos.

Esta interrelación disciplinar constituye el núcleo de la innovación surgida en el Oeste de los Estados Unidos de Norteamérica, la cual se conforma a partir de la diversidad y es producto de lo que juntos son capaces de hacer. Durante ese proceso, se aplican numerosas técnicas para establecer puentes en el lenguaje interdisciplinar complementado por métodos, con el objetivo de cruzarlos, integrarlos, mas no eliminarlos. Gold concluye que al menos ha encontrado más de un profesional con, al menos, un pie en uno de los cuatro cuadrantes de la matriz creativa. Eso nos debería conducir a una reflexión profunda en el proceso constructivo de los perfiles profesionales del futuro para un mundo complejo, incierto, ambiguo y volátil.

Es indispensable en la construcción de nuevo conocimiento, debido a la complejidad y volatilidad de las emergencias diarios y desafíos que nos presenta el mundo, tener la apertura a aceptar la diversidad y el trabajo en equipo como una manera de abordaje más eficaz y eficiente frente a los problemas y situaciones de la realidad local o global. Si construimos perfiles más interdisciplinares, lo que vamos a obtener como resultado es una transdisciplinariedad capaz de sortear obstáculos de mayor complejidad.

Gold (2007), afirma que géneros como el rock and roll resultan diferentes a la música country, lo mismo que los nuevos géneros que surgen, como por ejemplo el punk rock. También sostiene que los nuevos medios ayudan al surgimiento de nuevos géneros. Mientras que existe una diferencia notable entre medios y contenidos, se entiende que un medio puede albergar múltiples géneros y que un género puede cruzar múltiples medios. Y esto está sucediendo cada vez más frecuentemente.

Por ejemplo, si eres una empresa que fabrica impresoras puede llevarte quizás cinco años fabricar, diseñar y ensamblar una nueva impresora, sin embargo, si como compañía quisieras moverte hacia adelante en el negocio de la documentación y preguntar, ¿qué nuevos géneros de impresoras necesita el futuro?, ¿cómo crear un nuevo género? No puedes hacerlo de manera abstracta, tampoco escribiendo un documento académico, para expresarlo en otras palabras, no puedes crear rock and roll sin grabar una canción de rock.Y es de esa manera, que urge la necesidadde científicos, ingenieros, diseñadores y artistas, todos trabajando juntos por un género cuyo resultante será la combinación de todas esas fuerzas creativas. (Gold, 2007, p.76)

Finalmente, siguiendo a Gold, la lectura es una de las dos formas primarias de comunicarse entre los seres humanos e intercambiar información y conocimiento. Por supuesto, la otra forma es el diálogo. A diferencia del lenguaje oral, la lectura se mantiene y prevalece en el tiempo. Ahora, la lectura ha evolucionado y ha sufrido cambios con el tiempo, se ha redefinido: cada superficie se convierte en legible. Basta con ver la televisión digital, las pantallas de los ordenadores, las plataformas digitales o las vallas publicitarias digitales en la ciudad. Los textos se mueven, cambian y actualizan. Existen cada vez más medios diferentes y, también, distintas formas de lectura de los mismos. Cada una de ellas posee su propio significado. 
A partir de esta reconfiguración, esta investigación propone la plataforma de pensamiento y acción CITAD o por sus siglas en inglés: SETAD, que transforma la aproximación a la resolución de problemas o identificación de oportunidades a conformarse en una transdisciplina que reconfigura, modela, experimenta, soluciona, al contener en ella, la ciencia (science), la ingeniería (engineering), la tecnología (technology), el arte (art) y el diseño (design).

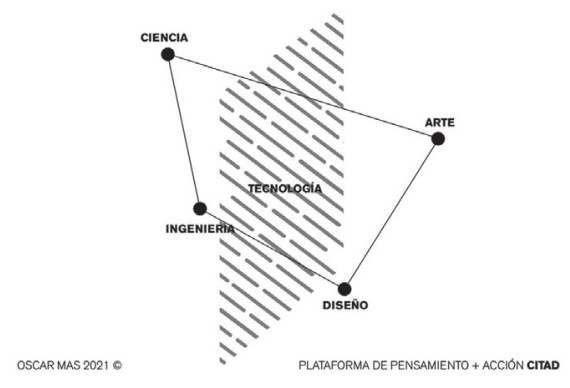

El esquema propuesto muestra los cuatro ejes que se desplazan en el espacio, en forma libre, generando tensiones positivas y de aprendizaje, catalizados por la tecnología como plataforma para potenciar el intercambio, la interrelación y, por supuesto, los productos resultantes de esas interacciones.

\section{Notas}

1. Faceplate o placa frontal, trata sobre una pieza de máquina, o interruptor o salida eléctrica.

2. Eugene A. Nida, lingüista estadounidense considerado el padre de la teoría de la equivalencia dinámica y formal en la traducción de la Biblia.

\section{Referencias}

Arte, Ciencia, Diseño e Ingeniería: Los cuatro sombreros de Rich Gold. Seisdeagosto.com. (2021). Retrieved 3 May 2021, from https://www.seisdeagosto.com/indica/2008/09/200809-arte-ciencia-diseno-e-ingenieria-los-cuatro-sombreros-de-rich-gold/.

Burgos, J. (2009). Arte, Ciencia, Tecnología y Diseño. Diseño como evidencia de la simbiosis entre Arte, Ciencia y Tecnología. Revista de arte y estéticacontemporánea.

Gold, R. (2007). The Plenitude: Creativity, Innovation and Making Stuff. MIT press. 


\begin{abstract}
The interaction between the various disciplines is a two-way relationship, where all of them are nurtured, defined, re-defined and their disciplinary boundaries are increasingly blurred, due to this permanent reconfiguration. Human beings are exposed to a variety of situations, uncertain and complex, and it is precisely these challenges that allow exercising not only interpretation, but also action to solve them, through the elaboration of solution hypotheses and then submitting them to a experimentation process to contrast and obtain conclusions that allow solving these challenges. This complex process, not only cognitive, but also oriented towards solving a problematic reality, enhances acuity in observation, reflection and problem-solving capacity. Based on this reconfiguration, this paper proposes the CITAD platform for thought and action or for its acronym in English: SETAD, which transforms the approach to solving problems or identifying opportunities, forming a transdiscipline that reconfigures, models, experiments, solves the question, by containing in it, Science (Science), Engineering (Engineering), Technology (Technology), Art (Art) and Design (Design).
\end{abstract}

Keywords: Design - art, science - engineering - technology - platform - thinking - design epistemology.

Resumo: A interação entre as várias disciplinas é uma relação de mão dupla, onde todas elas são nutridas, definidas, redefinidas e seus limites disciplinares são cada vez mais borrados, devido a essa reconfiguração permanente. O ser humano está exposto a uma variedade de situações, incertas e complexas, e são precisamente estes desafios que nos permitem exercer não só a interpretação, mas também a ação para as resolver, através da elaboração de hipóteses de solução e depois submetê-las a um processo de experimentação. Contrastar e obter conclusões que permitam resolver esses desafios. Este processo complexo, não só cognitivo, mas também orientado para a resolução de uma realidade problemática, potencia a acuidade de observação, reflexão e resolução de problemas. A partir dessa reconfiguração, este artigo propõe a plataforma de pensamento e ação do CITAD ou por sua sigla em inglês: SETAD, que transforma a abordagem de resolução de problemas ou identificação de oportunidades, formando uma transdisciplina que reconfigura, modela, experimenta, resolve a questão, ao conter nele, Ciência (Ciência), Engenharia (Engenharia), Tecnologia (Tecnologia), Arte (Arte) e Design (Design). Palavras chave: Design, arte, ciência, engenharia, tecnologia, plataforma, pensamento, epistemologia de design.

Palavras-chave: Design - arte - ciência - engenharia - tecnologia - plataforma - pensamento - epistemologia do design.

[Las traducciones de los abstracts fueron supervisadas por el autor de cada artículo] 